\title{
The History of Dengue in the United States and its Recent Emergence
}

\author{
Coreen Beaumier • Melissa N. Garcia • Kristy O. Murray
}

Published online: 22 January 2014

(C) Springer International Publishing AG 2014

\begin{abstract}
Dengue virus (DENV) is the most common and widespread vector-borne arbovirus in the world, and over the past 30 years, there has been a dramatic increase in incidence and geographic spread. Considering the difficulty of preventing infections and consequence of severe disease, it is important to reflect on the history of dengue in the United States and focus attention on its recent re-emergence. In this paper, we report the historical significance of dengue outbreaks in the USA between 1780 and 1945, along with the elimination strategies of the vector mosquito, Aedes aegypti. We then detail the re-emergence of dengue into the USA between 1999 and today and discuss future strategies for detection and control.
\end{abstract}

Keywords Viral tropical medicine $\cdot$ Dengue virus (DENV) · Dengue $\cdot$ Virus $\cdot$ Vector-borne arbovirus $\cdot$ Mosquito $\cdot$ United States · Tropical medicine

\section{Dengue Virus}

Dengue virus (DENV), often known as "breakbone fever," is the most common and widespread vector-borne arbovirus in the world, with an estimated 390 million infections annually resulting in 96 million clinical cases of disease [1]. Over the past 50 years, there has been a 30 -fold increase in the incidence of dengue worldwide [2]. Dengue is transmitted by Aedes (Ae.) species mosquitoes, and humans are the primary reservoir of infection and contribute to the geographic spread [3]. There are four serotypes of DENV (DENV-1, DENV-2,

C. Beaumier $\cdot$ M. N. Garcia $\cdot$ K. O. Murray $(\bowtie)$

Department of Pediatrics, Section of Pediatric Tropical Medicine, National School of Tropical Medicine, Baylor College of Medicine and Texas Children's Hospital, 1102 Bates St., Suite 550, Houston, TX 77030, USA

e-mail: kmurray@bcm.edu
DENV-3, and DENV-4), with infection with one serotype not conferring long-term protection from the others. In fact, subsequent infection with a different serotype increases the risk for severe disease and hemorrhagic manifestations [3].

Infection with DENV can result in a wide spectrum of clinical manifestations, ranging from subclinical to severe disease, including death [4]. In 2009, the World Health Organization (WHO) published new case definitions to aid in the diagnosis of clinical dengue infections [2]. A probable case of dengue fever is defined as fever plus two or more of the following symptoms: (1) anorexia and nausea/vomiting, (2) rash, (3) aches and pains including retro-orbital pain, (4) leukopenia, (5) positive tourniquet test, or (6) warning signs. Warning signs, which indicate a higher probability of developing severe dengue, are defined as: (1) abdominal pain or tenderness, (2) persistent vomiting, (3) clinical fluid accumulation, (4) mucosal bleeding, (5) lethargy/restlessness, (6) liver enlargement, and/or (7) increase in hematocrit concurrent with rapid decrease in platelets. Severe dengue is defined as having one or more of the following signs: (1) plasma leakage and/or fluid accumulation which may lead to shock [dengue shock syndrome], (2) hemorrhagic manifestations [dengue hemorrhagic fever], and/or (3) severe organ impairment [2].

At this time, there is no specific treatment other than supportive care for clinical dengue infections, nor is there any commercially available vaccine to prevent infection. Prevention of disease is focused on reducing exposure to mosquitoes, both through personal protective measures and reduction of mosquito populations by emptying containers and other water collection sites that provide suitable breeding habitats [2]. Considering the difficulty of preventing infections, severity of disease from infection, and the ever-expanding geographic range and incidence of infection globally, it is important to reflect on the history of dengue in the United States and focus attention on its recent re-emergence. 


\section{History of Dengue in the United States}

Dengue was first described in the USA by Benjamin Rush in Philadelphia in 1780, and though the causative agent was not identified, the clinical presentations were compatible with classic dengue fever [3]. Dengue soon began to continually show itself in the southern USA, beginning 47 years later, in 1827, when epidemics of suspected dengue occurred in some of its southern cities, including Charleston (South Carolina), Savannah (Georgia), Pensacola (Florida), and New Orleans (Louisiana). Reports from these epidemics cited not only classic dengue symptoms but also minor hemorrhagic manifestations such as bleeding from the gums, throat, and bowels in addition to obstetric complications such as miscarriage and pre-mature births [5]. In 1850, Mobile (Alabama), Augusta (Georgia), and again the cities Charleston, New Orleans, and Savannah experienced more epidemics attributed to dengue, with clinical presentations similar to those seen in 1827 . Interestingly, those who were sick in 1827 appeared to be immune to the contagion in 1850 , suggesting that the pathogen was the same [5]. It is important to note, however, that clinical data presented by Carey et al. in 1971 suggests that these epidemics may have potentially been due to the alphavirus chikungunga and not dengue as originally believed [6]. Dengue, chikungunga, and other viral illnesses can often be difficult to differentiate clinically without a diagnostic test.

The $1870 \mathrm{~s}$ saw two major outbreaks attributed to a denguelike syndrome. In 1873, 40,000 cases were reported in New Orleans, and another more widespread epidemic in 1879-1880 affected New Orleans, Savannah, Augusta, and Charleston [5]. These cases were different from the previous epidemics of 1827 and 1850 in that no pre-existing immunity was observed, although similar clinical manifestations were reported [5]. By 1885, dengue began to spread westward into Texas, and between 1885 and 1886 it was responsible for 16,000 clinical cases in Austin, which was nearly three-quarters of the city's population at the time. It soon spread to the surrounding railroad towns and then throughout the rest of the state by 1897 . In 1898-1899, outbreaks occurred in both Florida and Texas, most likely due to the movement of troops participating in military action occurring in Cuba [5]. Outbreaks also occurred in the Texas gulf coast cities of Houston, Galveston, and Brownsville in 1907 and again in 1918 with the compounding factor being once again military units travelling from endemic regions, this time returning from the West Indies [5].

Several years later in 1922, another pandemic emerged in the southern states of Texas, Louisiana, Florida and Georgia with 500,000 cases of presumed dengue in Texas and 30,000 in Savannah alone. During this epidemic, there was a reported increase in hemorrhagic symptoms but mortality remained low [5]. Twelve years later, in 1934, a dengue epidemic began in Miami and spread northward up into Georgia. Those affected in 1934 showed a milder clinical manifestation and pre- exposure to the 1922 outbreak did not confer immunity. Taken together, this information suggests that the DENV serotypes responsible for the 1922 and 1934 outbreaks were different [5]. The last historical outbreaks within the continental USA occurred in the gulf cities of Texas in 1941 and Louisiana in 1945 , with reported symptoms being clinically mild with no hemorrhagic manifestations or obstetric complications $[5,7]$. Outbreaks described above occurred primarily in hubs of mass transit: railroads, ports, and stations of military personnel, where the mass movement of people was the key to the spread of dengue.

In the absence of a vaccine, control of the vector $A e$. aegypti was critical in decreasing the burden of dengue in the Americas; however, the initial driving force for this mosquito's eradication was not dengue, but yellow fever virus [8, 9]. In 1901, William Gorgas, following Walter Reed and Carlos Finlay's lead, began the first effort to eradicate $A e$. aegypti from Havana, Cuba. Oswaldo Cruz soon followed in 1903 with similar methods in Rio de Janeiro, Brazil. Their primary means of killing the vector was a two-pronged attack comprised of fumigation and preventing the presence of standing water via open containers $[8,9]$. The Brazilian government, in partnership with the New York Based Rockefeller Foundation, began a mass eradication campaign against $A e$. aegypti and was successful in local campaigns, as were other countries such as Paraguay, Colombia, Bolivia, Peru, and Ecuador $[8,9]$. In 1947, the Pan American Sanitary Bureau (PASB) took specific interest in the effort and with the advent of DDT the goals of eradication spread throughout the continent of South America [8,9]. Although the efforts of the PASB were deemed successful, the vector still remained in the Caribbean, Cuba, United States, and Venezuela. PASB's efforts persisted through the early 1960s, after which its activity declined, most likely from decreased political interest, cost, vector resistance to DDT, and lack of public support [8]. This decline in eradication campaigns has contributed to global re-infestation with Ae. aegypti and re-emergence of dengue [8]. Yellow fever has not re-emerged presumably due to an effective vaccine.

\section{Recent Emergence of Dengue in the United States}

For more than 30 years, it was believed that dengue had been eliminated from the Americas [10], until 1977-1979 when dengue reappeared in the Caribbean and Mexico [11, 12]. Due to the geographic expansion of DENV, passive surveillance was instituted in Texas in 1980 to detect possible cases of DENV infection [10]. During that year, 63 cases of DENV were identified, with $43 \%$ of cases acquiring their infection on the USA side of the border. Autochthonous cases were identified in six counties, mostly in the southern part of the state along the border [10]. 
In 1999, an outbreak of DENV in Nuevo Laredo, Tamaulipas, Mexico spilled over across the Rio Grande River into Laredo, Texas, with two autochthonous cases confirmed (Fig. 1) [13]. Interestingly, none of the cases were diagnosed with dengue at the time of illness, and all were identified through retrospective chart review with participating hospitals and clinics, highlighting concerns with physician identification of dengue cases.

Between 2001 and 2002, an outbreak of DENV was confirmed in Hawaii, which was the first time the virus had been identified on the islands in 57 years [14]. During the outbreak, 122 cases were confirmed, with DENV-1 identified as the circulating serotype. It was believed the virus was introduced by an infected traveler returning from Tahiti in French Polynesia. Interestingly, no Ae. aegypti mosquitoes were identified in Hawaii at the time, and it was the first time a dengue outbreak was solely attributed to the vector $A e$. albopictus, a highly prevalent mosquito present in North America that was first introduced in the 1980s via the importation of tires [15].

In the early part of the twenty-first century, additional outbreaks occurred in Texas with evidence of autochthonous transmission. In 2005, a DENV-2 outbreak was confirmed in
Brownsville, Texas along the US-Mexico border [16]. A total of 24 cases were identified, including three with no history of travel into Mexico. One of these cases developed dengue hemorrhagic fever. A seroepidemiological survey was conducted following the outbreak, and investigators found that $4 \%$ of Brownsville residents had evidence of recent dengue infection compared with $32 \%$ of residents across the border in Matamoros, Mexico [17].

Similar to the Laredo, Texas [13], dengue was not considered as part of the differential diagnosis in the hemorrhagic fever case in Brownsville. Investigators in Houston, Texas were also concerned about underdiagnosis of dengue in the region, and as a consequence, they conducted a retrospective study to retest all specimens for DENV that were submitted between 2003 to 2005 for West Nile virus testing [18]. Using this approach, the investigators identified 47 cases positive for acute DENV, with only two cases having a history of travel to an endemic area. Further examination of the positive cases revealed that two were positive for DENV-2 by polymerase chain reaction (PCR), and none of the 47 cases had dengue listed as a possible cause of the illness. These recent outbreaks in Texas highlight the concern of disease recognition by health care providers.

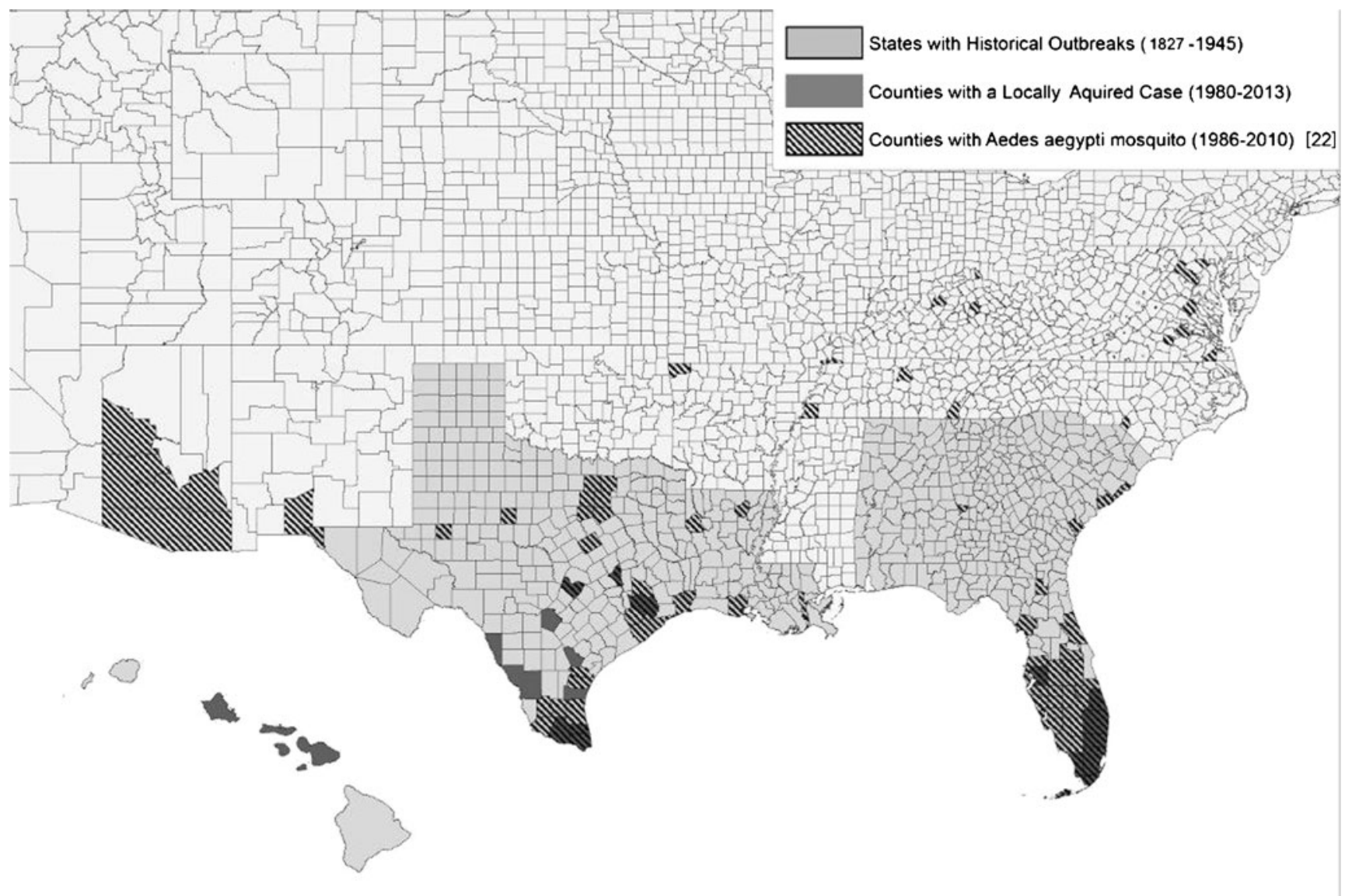

Fig. 1 Distribution of dengue fever outbreaks and Aedes aegypti mosquitoes in the United States 
Besides evidence of transmission in Texas over the past decade, DENV has also recently emerged in Florida. In 2009 in Key West, Florida, three cases of dengue were identified [19], and a seroepidemiological study identified 13 additional cases of recent DENV infection, equating to $5.4 \%$ of the population. Between 2009 and 2010, enhanced and active surveillance identified a total of 28 cases in the Florida Keys [20]. According to the ArboNet surveillance system of the Centers for Disease Control and Prevention (CDC) [21], locally acquired dengue cases are continuing to be reported, with 58 cases in Florida in 2010, 7 Florida cases and 4 Hawaii cases in 2011, 2 Florida cases in 2012, and, as of November 19, 2013, 20 Florida cases and 3 Texas cases reported.

\section{Future Strategies for Prevention and Control}

With competent mosquito vectors throughout the southern USA and lack of a preventative vaccine, we can expect to continue to observe periodic outbreaks of dengue in high risk areas for transmission as shown in Fig. 1. Public health strategies will need to focus on several important areas for diagnosis, prevention, and control. First, as highlighted by the outbreaks in Texas, we need to prioritize education of health care providers in the identification and diagnosis of dengue cases. Second, we must strengthen both active and passive surveillance of clinical cases in areas where competent vectors are prevalent. Third, we must streamline diagnostic testing to ensure timely diagnosis and public health response to emerging epidemics. Fourth, we must identify factors at the local level that can contribute to emergence, including dense populations, areas of poverty, substandard housing, and containers conducive to breeding of $A e$. species mosquitoes. Finally, we must prioritize research towards rapid diagnostics as well as effective treatment and vaccination strategies. Together these steps can strengthen the detection and control of dengue in the USA.

\section{Compliance with Ethics Guidelines}

Conflict of Interest Coreen Beaumier, Melissa N. Garcia, and Kristy O. Murray declare that they have no conflict of interest.

Human and Animal Rights and Informed Consent This article does not contain any studies with human or animal subjects performed by any of the authors.

\section{References}

1. Bhatt $\mathrm{S}$ et al. The global distribution and burden of dengue. Nature. 2013;496(7446):504-7.

2. World Health Organization. Dengue: guidelines for diagnosis, treatment, prevention, and control. 2009. Available from: http:// whqlibdoc.who.int/publications/2009/9789241547871_eng.pdf.

3. Gubler DJ. Dengue and dengue hemorrhagic fever. Clin Microbiol Rev. 1998;11(3):480-96.

4. Kalayanarooj S. Clinical manifestations and management of dengue/ DHF/DSS. Trop Med Health. 2011;39(4 Suppl):83-7.

5. Ehrenkranz NJ et al. Pandemic dengue in Caribbean countries and the southern United States-past, present and potential problems. N Engl J Med. 1971;285(26):1460-9.

6. Carey DE. Chikungunya and dengue: a case of mistaken identity? J Hist Med Allied Sci. 1971;26(3):243-62.

7. Hayes G, Scheppf PP, Johnson EB. An historical review of the last continental U.S. epidemic of dengue. Mosq News. 1971;31:422-27.

8. Brathwaite Dick O et al. The history of dengue outbreaks in the Americas. Am J Trop Med Hyg. 2012;87(4):584-93.

9. Pinto Severo O. Campaign of eradication of Aedes aegypti in the Americas; its organization, development and results since December 1954. Bol Oficina Sanit Panam. 1955;38(4):378-98.

10. Hafkin B et al. Reintroduction of dengue fever into the continental United States. I. Dengue surveillance in Texas, 1980. Am J Trop Med Hyg. 1982;31(6):1222-8.

11. Pan American Health Organization. Dengue in the Caribbean, 1977. Washington DC: PAHO; 1979.

12. Centers for Disease Control. Follow-up on dengue-Mexico. Morb Mortal Wkly Rep. 1980;129:169-70.

13. Centers for Disease Control and Prevention. Underdiagnosis of dengue-Laredo, Texas, 1999. Morb Mortal Wkly Rep. 2001;50:57-9.

14. Effler PV et al. Dengue fever, Hawaii, 2001-2002. Emerg Infect Dis. 2005;11(5):742-9.

15. Moore CG, Mitchell CJ. Aedes albopictus in the United States: tenyear presence and public health implications. Emerg Infect Dis. 1997;3(3):329-34.

16. Centers for Disease Control and Prevention. Dengue hemorrhagic fever-U.S.-Mexico border, 2005. Morb Mortal Wkly Rep. 2007;56: 785-9.

17. Ramos MM et al. Epidemic dengue and dengue hemorrhagic fever at the Texas-Mexico border: results of a household-based seroepidemiologic survey, December 2005. Am J Trop Med Hyg. 2008;78(3):364-9.

18. Murray KO et al. Identification of dengue fever cases in Houston, Texas, with evidence of autochthonous transmission between 2003 and 2005. Vector Borne Zoonotic Dis. 2013;13(12):835-45.

19. Radke EG et al. Dengue outbreak in Key West, Florida, USA, 2009. Emerg Infect Dis. 2012;18(1):135-7.

20. Centers for Disease Control and Prevention. Locally acquired dengue-Key West, Florida, 2009-2010. Morb Mortal Wkly Rep. 2010;59:577-81.

21. Centers for Disease Control and Prevention. ArboNet surveillance data. Atlanta: CDC; 2013.

22. Eisen L, Moore CG. Aedes (Stegomyia) aegypti in the continental United States: a vector at the cool margin of its geographic range. J med Entomol. 2013;50:467-78. 